\title{
UMA ABORDAGEM INTERDISCIPLINAR PARA O ENSINO EM ENGENHARIA: RELATO DE UMA EXPERIÊNCIA
}

Michele OtobelliBertéli-mobertel@ucs.br

Carlos Alberto Costa-cacosta@ucs.br

CesarAugusto Bernardi-cabernar@ucs.br

Universidade de Caxias do Sul

Rua Francisco Getúlio Vargas, 1130

CEP 95070-560 - Caxias do Sul - Rio Grande do Sul

Resumo: Este artigo apresenta o resultado de um trabalho de intervenção sobre o processo de ensino interdisciplinar em disciplinas dos cursos de engenharias. O trabalho foi desenvolvido por meio de um estudo de caso realizado em quatro disciplinas dos cursos de engenharia: produção, controle e automação, civil, elétrica e no curso de arquitetura. Foi utilizada uma abordagem exploratória e descritiva com caráter qualitativo. O tema central do estudo foi o desenvolvimento de um projeto elétrico, guiado pelos conceitos de gestão de projetos e com uma análise de experimentos como forma de avaliar os resultados do trabalho. Os resultados mostraram que a integração de conteúdo dentro de diferentes disciplinas que extrapolam as fronteiras tradicionais dos cursos de graduação é possível. Como retorno por parte dos estudantes envolvidos houve a sinalização positiva pela oportunidade de aplicação dos conceitos desenvolvidos em cenários de outras turmas do mesmo semestre. Futuros estudos são necessários no sentido de estratificar em mais detalhes a amostra para avaliar o nível de assimilação e aprendizagem, além das notas finais obtidas pelos alunos.

Palavras-chave: Interdisciplinaridade. Projeto. CANVAS.

\section{INTRODUÇÃO}

A discussão sobre alternativas para potencialização do conhecimento nos bancos universitários motivou o desenvolvimento de uma pesquisa prática com o tema de interdisciplinaridade envolvendo três diferentes disciplinas nos cursos de engenharia da Universidade de Caxias do Sul (UCS). O interesse pela pesquisa se deu com possibilidade de investigar o efetivo resultado da aplicação e utilização de novas estratégias de aprendizagem pelos estudantes dessas turmas.

Ressalta-se que o objetivo central da proposta de intervenção é verificar se existe diferença significativa no resultado de aprendizagem obtido pelos estudantes envolvidos no desenvolvimento de um projeto elétrico, considerando as orientações recebidas quanto a fase inicial de planejamento do escopo deste projeto. Nesse sentido, a proposta adotou como linha de estudo a abordagem baseada em problemas (RIBEIRO; ESCRIVÃO-FILHO; MIZUKAMI, 2004), em que, nas três turmas, os alunos foram desafiados com cenários complementares fazendo uso da estratégia de ensino jogo de papéis ou role-play (SOUZA; CASA NOVA, 2019) em que cada turma assume um papel no processo. Além do objetivo norteador da proposta de intervenção, pode-se citar ainda, como benefícios identificados para os estudantes de cada turma específica, os seguintes: 
a) Turma de Gerenciamento de Projetos (PRO0206): consolidação dos conhecimentos teóricos associados à construção de escopo de projetos, com base na ferramenta CANVAS de projeto, por meio da atividade de avaliação e crítica de planos de projeto considerando o escopo;

b) Turma de Instalações Elétricas Prediais (CIV0212): oportunidade de melhoria contínua durante a fase de desenvolvimento do projeto, por meio de feedback da turma PRO0206 e recebimento de orientação específica sobre o conceito de escopo de projeto;

c) Turma de Planejamento e Avaliação de Experimentos (PRO0207): realização da atividade prática proposta na disciplina com uso de dados reais e elaboração do projeto, da fase de planejamento e coleta de dados até a aplicação de análises estatísticas.

A próxima seção apresenta a fundamentação teórica utilizada para a execução do projeto, seguida pela apresentação do método empregado e dos resultados encontrados.

\section{FUNDAMENTAÇÃO TEÓRICA}

A proposta para a realização deste trabalho considera que o ambiente de aprendizagem não se limita nas paredes da sala de aula, nem em um conjunto de disciplinas pré-estabelecidas de um semestre, ou muito menos numa abordagem unidirecional professor-aluno dentro de uma estrutura de governamento curricular (FOUCAULT, 2011). O ambiente de aprendizagem deve considerar a multiplicidade dos conteúdos e cenários em que, de alguma forma, conteúdos de diferentes disciplinas, lecionadas de forma concomitante, se complementam durante o processo de aprendizagem. Desta forma tal ambiente deve estar aberto a experimentação curricular oportunizando experiências que venham a contribuir para a aprendizagem (LARROSA, 2002; FERRARO, 2017). Com base nisso, a presente proposta de intervenção usa o conceito de interdisciplinaridade, entre disciplinas, como uma forma de alavancar, consolidar e assimilar o conhecimento (LÜCK, 2000; PAVIANI, 2008; FAZENDA, 2011). Demo (1997, p. 88) define interdisciplinaridade como "a arte do aprofundamento com sentido de abrangência, para dar conta, ao mesmo tempo, da particularidade e da complexidade do real. A motivação para tal está na questão de o quanto os conteúdos de uma disciplina realmente facilitam os estudantes no processo de assimilação e fixação de novos conceitos, ou seja, o clássico princípio do prérequisito vinculado a uma disciplina. De forma complementar, é difícil a realização desse tipo de projeto senão por meio de abordagem de trabalho em equipes fazendo uso de técnicas como trabalhos em grupo (ROSA; BARCELOS, 2005; CAMARGO; OLIVEIRA, 2019).

Em projetos de engenharia, dois aspectos são normalmente citados como áreas de competência e domínio: o aspecto técnico e o aspecto de gestão de projeto (BAZZO; PEREIRA, 2013). No aspecto técnico, é valorizada a competência técnica do engenheiro em buscar a melhor solução possível, dados um determinado cenário e conjunto de restrições (MADUREIRA, 2010). No aspecto de gestão, é considerada a competência em saber conduzir o projeto dentro dos prazos, conjugando as diferentes atividades e considerando os recursos disponíveis (GIDO; CLEMENTS; BAKER, 2018). Esses dois aspectos são normalmente organizados curricularmente em disciplinas diferentes, que podem não possuir pré-requisitos umas das outras, e que não são necessariamente realizadas pelos estudantes no mesmo semestre. Esse contexto apresenta uma oportunidade de repensar o ensino, fazendo uso de abordagens interdisciplinares; pois considerando a forma de aprendizagem do aluno atual, não há como impor uma ordem cronológica dentro de um currículo tradicional (HUYGHE; TOTTÉ; VERHAGEN, 2013). Assim, esse trabalho fez uso dessa base para apoiar o experimento proposto que segue apresentado na próxima seção. 


\section{CONTEXTO DA INTERVENÇÃO E INDICADORES}

O projeto de intervenção ocorreu na Universidade de Caxias do Sul (UCS), na Área de Conhecimento de Ciências Exatas e Engenharias da Cidade Universitária, tendo como cenário o ambiente de ensino e aprendizagem de três disciplinas, que foram lecionadas no mesmo semestre, e que envolvem, curricularmente, os cursos de engenharia de produção, engenharia civil, engenharia elétrica e o curso de arquitetura. A seguir são apresentadas as disciplinas.

a) Gerenciamento de Projetos (PRO0206): vinculada aos cursos de Engenharia de Produção, Civil, Mecânica, Elétrica e Controle e Automação, é ofertada regularmente no nono semestre dos cursos. É comum que estudantes de outros cursos selecionem essa disciplina para cursarem como optativa ou eletiva. O principal objetivo da disciplina é propiciar ao estudante a compreensão sobre os fundamentos básicos da gerência de projetos, destacando o desenvolvimento da capacidade e do uso de ferramentas para a definição do escopo de um projeto. A disciplina faz uso da estratégia de Aprendizagem Baseada em Projetos, em que os alunos trabalham sobre um projeto escolhido por eles, enquanto equipe, e a cada etapa do planejamento do projeto são assimilados progressivamente novos conceitos e conhecimentos;

b) Instalações Elétricas Prediais (CIV0212): cursada pelos estudantes da Engenharia Civil e Arquitetura e Urbanismo e está prevista no oitavo semestre do currículo. Tem como objetivo capacitar os alunos a elaborar e interpretar um projeto de instalações elétricas prediais em baixa tensão, visando a materialização das construções e dominando todos os componentes dela; relacionar o projeto elétrico predial com o projeto arquitetônico; dimensionar as instalações elétricas prediais; detalhar o projeto elétrico predial e apresentar noções de redes prediais e urbanas. Entendendo que, ao final do curso, o egresso possui esta atribuição conferida a ele pelas entidades de classe, percebe-se a clara necessidade de capacitar estes profissionais com um arcabouço de ferramental suficiente que lhe forneça segurança no desempenho da atividade;

c) Planejamento e Avaliação de Experimentos (PRO0207): ofertada no nono semestre do curso de Engenharia de Produção. O objetivo da disciplina é propiciar ao aluno conhecimentos para entender, aplicar e interpretar os diversos tipos de projetos de experimentos, usando estatística ANOVA, Fatorial e outras técnicas para análise e melhoria de processos. Ao longo do tempo, foram sendo propostas mudanças na metodologia da disciplina, buscando que o estudante entenda a aplicação das ferramentas, mas principalmente sinta-se encorajado a aplicar em sua vida profissional o uso da estatística como estratégia para tomada de decisão bem como mudanças nos processos que tragam maior eficiência à operação.

$\mathrm{O}$ Quadro 1 resume as informações referentes às turmas envolvidas no projeto de intervenção, que foi realizado no segundo semestre de 2019. Destaca-se o aspecto interdisciplinar das disciplinas e os cursos em que estão formalmente alocadas. A disciplina de Gerenciamento de Projetos que atende diferentes cursos das áreas das engenharias (Produção, Civil, Elétrica, Controle e Automação, e outros). A disciplina de Instalações Elétricas Prediais atende a dois cursos em específico (Civil e Arquitetura), e a turma de Planejamento e Avaliação de Experimentos atende somente o curso de Engenharia de Produção.

Quadro 1 - Caracterização das turmas participantes do projeto em 2019

\begin{tabular}{|c|c|c|c|}
\hline Disciplina & PRO0206 & CIV0212 & PRO0207 \\
\hline Turma & A & A & A \\
\hline Dia da oferta & Segunda-feira & Quinta-feira & Quinta-feira \\
\hline Horário da oferta & $19: 40$ às $22: 35$ & $19: 40$ às $22: 35$ & $19: 40$ às $22: 35$ \\
\hline Quantidade de alunos & 28 & 29 & 22 \\
\hline
\end{tabular}

Fonte: Autores (2020) 


\section{MÉTODO PROPOSTO}

A proposta de intervenção foi planejada no primeiro semestre de 2019, sendo ajustada a execução dos planos de ensino de forma a permitir, além da interdisciplinaridade, a sincronia de conteúdos entre as disciplinas. A aplicação ocorreu no segundo de semestre de 2019.

Para todas as disciplinas, durante o primeiro encontro, os professores expuseram o contexto do projeto de intervenção e seu caráter avaliativo, orientando quanto a forma que o experimento seria conduzido. Isso permitiu a sensibilização dos alunos quanto ao comprometimento com a atividade.

O objetivo geral da proposta de intervenção foi verificar se há diferença significativa nos resultados de aprendizagem entre dois grupos (A e B) de alunos da disciplina de Instalações Elétricas Prediais (CIV0212), em função da intervenção realizada, com e sem orientação para a criação de um CANVAS de projeto, como forma de orientar o planejamento e desenvolvimento do projeto da disciplina. A diferença foi mensurada por meio das notas alcançadas pelos alunos da turma CIV0212. O CANVAS de Projeto é uma ferramenta de planejamento, que contém as principais informações sobre um projeto estruturado na forma de um quadro (mapa) dividido em alguns blocos, que normalmente são preenchidos de forma conjunta pela equipe de projeto. Isso propicia melhor comunicação, alinhamento e desempenho na definição do projeto (FINOCCHIO JUNIOR., 2013).

A Figura 1 mostra, de forma esquemática, o processo conduzido pelo experimento envolvendo as três turmas. O projeto de intervenção interdisciplinar foi separado em três fases, sendo a fase 1 a formação dos grupos e recebimento (ou não) da orientação sobre projeto CANVAS. A fase 2 é composta pela execução da primeira fase do projeto elétrico com correção do CANVAS e a fase 3 pela avaliação dos resultados obtidos.

Figura 1 - Etapas da proposta de projeto de Intervenção.

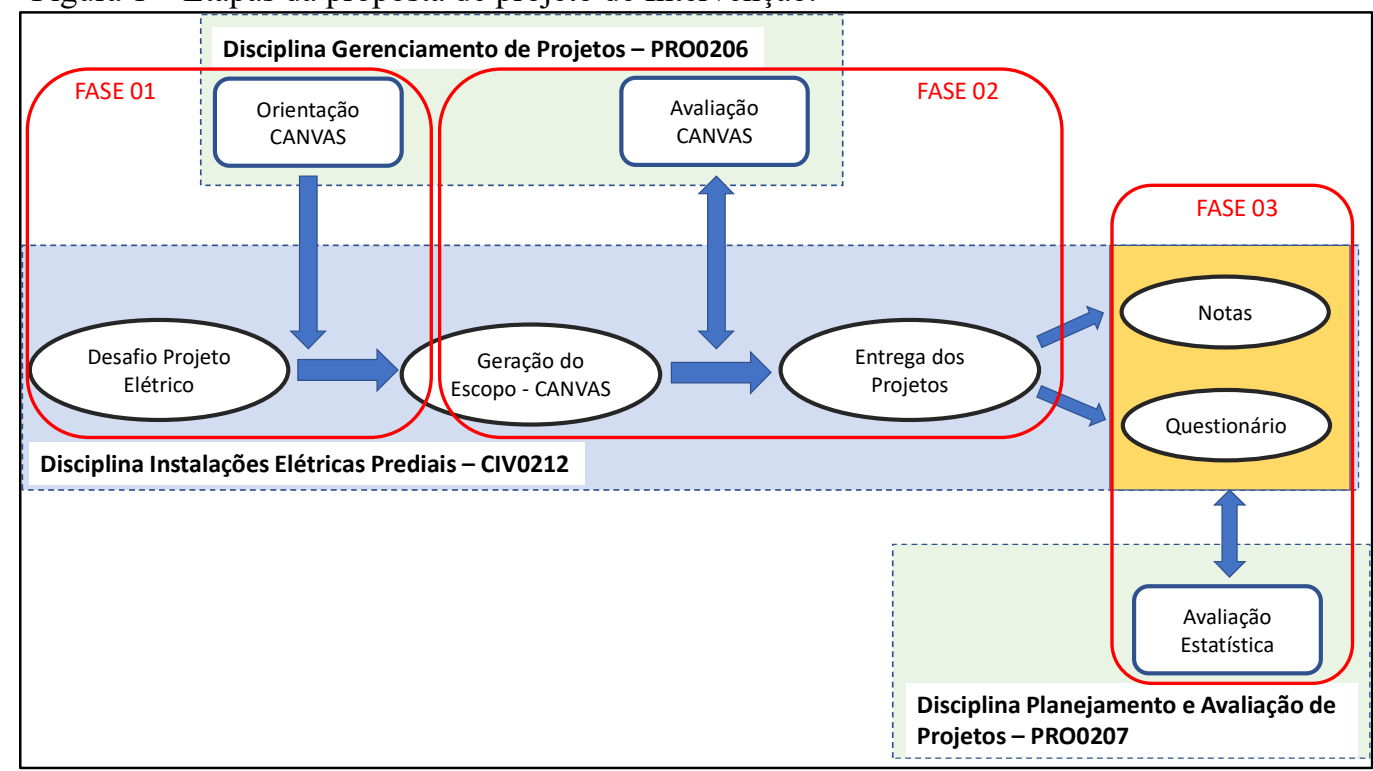

Fonte: Autores (2020)

Na fase 1, o professor da disciplina Instalações Elétricas Prediais (CIV0212) apresentou o teor dos requisitos a serem cumpridos para a elaboração do projeto elétrico de uma instalação para todos os alunos. A turma foi dividida em 9 equipes, que posteriormente foram alocadas em dois grupos (A e B). O grupo A, com quatro equipes, recebeu as orientações tradicionais da 
disciplina quanto ao processo de desenvolvimento do projeto estipulado. $\mathrm{O}$ grupo $\mathrm{B}$, com cinco equipes, recebeu as orientações do professor da disciplina de Gerenciamento de Projetos, também quanto ao processo de desenvolvimento do projeto estipulado, mas fazendo o uso da ferramenta CANVAS de projeto.

$\mathrm{Na}$ fase 2 os estudantes da turma de Gerenciamento de Projetos (PRO0206) receberam os projetos das equipes pertencentes ao Grupo B e realizaram a avaliação dos CANVAS gerados. $\mathrm{O}$ resultado foi compilado e retornado as equipes pertencentes ao Grupo B.

Por fim, na fase 3 foram realizadas as análises estatísticas, por parte dos alunos da turma de Planejamento e Avaliação de Experimentos (PRO0207), identificando as diferenças entre os resultados atingidos pelos dois grupos de equipes.

\section{APLICAÇÃO DA PROPOSTA DE INTERVENÇÃO}

\subsection{Fase 1 - apresentação do projeto}

O desafio foi composto por projeto elétrico de um prédio com 7 pavimentos, incluindo 2 pavimentos de garagem, 15 apartamentos, sendo 3 por andar, hall de entrada, cisterna e casa de máquinas. Foi solicitado aos estudantes que cada equipe deveria prever as necessidades elétricas deste prédio, o projeto do sistema de aterramento elétrico e do sistema de proteção contra descargas atmosféricas para proteção do prédio.

As equipes receberam três datas para as entregas de resultados, esclarecidas durante a fase 1 do projeto. Na quinta semana de aula, com as nove equipes formadas, o professor da disciplina que recebeu a intervenção (CIV0212) explicou o projeto elétrico do semestre até o horário de intervalo. Após, a turma foi dividida em dois grupos de equipes (A e B). O grupo A, constituído de quatro equipes, recebeu as orientações tradicionais da disciplina quanto a entrega da primeira etapa do projeto (como tem ocorrido ao longo dos últimos semestres) pelo professor e, o Grupo $\mathrm{B}$, com cinco equipes, recebeu orientação sobre os elementos que são considerados necessários no detalhamento de um plano de escopo de um projeto e a forma como esses elementos podem ser estruturados em CANVAS de projeto pelo professor da disciplina PRO0206, que forneceu material de apoio para ser consultado em outros momentos.

A primeira entrega, o plano de escopo do projeto, ocorreu duas semanas após a intervenção nas equipes, ou seja, orientações quanto ao desenvolvimento dos projetos com e sem CANVAS. Nessa entrega deveriam estar presentes no plano de escopo os seguintes elementos: justificativa, objetivo, produto final, equipe, possíveis stakeholders, restrições e riscos.

\subsection{Fase 2 - análise crítica do CANVAS}

A disciplina de Gerenciamento de Projetos (PRO0206) foi ajustada quanto a sua sequência de conteúdos de forma que no primeiro mês de aulas os alunos tivessem os conteúdos relativo à fase de elaboração da declaração de escopo de projetos, por meio da familiarização com a ferramenta CANVAS (FINOCCHIO JUNIOR, 2013). O projeto de intervenção foi explicado para os alunos e, salientou-se como isso contribuiria para o processo de aprendizagem de cada um, além de fazer parte do processo de avaliação da disciplina.

Foram simuladas duas situações prévias para construção de CANVAS, em que os alunos foram instigados a uma discussão sobre o propósito e importância de cada um dos elementos componentes do CANVAS de projeto. Dois projetos de comum conhecimento dos alunos foram utilizados, sendo um deles a Semana Acadêmica e outro um estudo de caso fíctício. As discussões das avaliações foram realizadas de forma pública, com intervenção do professor, alinhando as interpretações de cada um dos elementos presentes no CANVAS. 
Na semana 8, as equipes de alunos da disciplina de Gerenciamento de Projetos receberam as propostas de CANVAS, conjuntamente com a proposta do trabalho da disciplina de Instalações Elétricas Prediais. Todas as equipes receberam as mesmas quatro propostas, mantendo o anonimato dos autores.

Optou-se por uma entrega de todos os CANVAS para cada equipe, pois isso propiciou um processo de análise crítica mais consistente, dentro do contexto proposto, além de um debate da turma sobre cada uma das propostas. As equipes de Gerenciamento de Projeto tiveram duas semanas para realizarem as análises que foram compiladas pelo professor da disciplina de Gerenciamento de Projetos retornando a turma de Instalações Elétricas como sendo um momento de revisão do escopo do projeto.

\subsection{Fase 3 - coleta e análise dos resultados}

Os estudantes da turma PRO0207 estudaram os conceitos de pesquisa quantitativa do tipo survey e elaboraram um questionário a ser aplicado aos estudantes da turma CIV0212 com objetivo de investigar a percepção deles sobre a condução do trabalho (projeto elétrico) e da relação com definição do escopo. O questionário foi analisado pelos professores que participaram do projeto de intervenção das três disciplinas mediante o objetivo da proposta.

Na semana 10, o questionário foi disponibilizado para a turma CIV0212, que teve 15 dias para retornar com as respostas, via formulário google. Essas respostas foram utilizadas para realizar análises estatísticas, juntamente com a nota final do projeto de cada grupo, o semestre do estudante em relação ao curso, frequência do estudante e seu curso de origem da turma CIV0212. Cada equipe da turma PRO0207 recebeu os critérios para realizar as análises estatísticas, conforme:

a) equipe 01: análise descritiva e ANOVA One-Way entre notas recebidas pelos estudantes dos Grupos A e B. Variável resposta $(\mathrm{y})=$ notas dos estudantes e variável $(\mathrm{x})$ = orientação sobre escopo da turma PRO0206;

b) equipe 02: análise descritiva e ANOVA Two-Way entre percepção dos estudantes Grupos A e B. Variável resposta $(\mathrm{y})=$ notas dos estudantes, variável $(\mathrm{x} 1)=$ alunos que receberam orientação sobre escopo e variável $(\mathrm{x} 2)$ = a orientação recebida sobre o CANVAS foi útil para a organização da equipe na execução da primeira etapa do projeto;

c) equipe 03: análise de regressão múltipla (questionário aplicado). A regressora empregada (y) foi a nota recebida pelos estudantes e todas as demais variáveis foram usadas no modelo com objetivo de identificar as que mais explicam a nota recebida;

d) equipe 04: projeto fatorial fazendo uso da variável resposta $(y)=$ notas dos estudantes e as variáveis x sendo as 5 primeiras mais significativas da análise de regressão múltipla obtida pela equipe 3 ;

e) equipe 05: análise de regressão múltipla usando como variável regressora $(y)=$ aprendi os conteúdos da disciplina.

Os dados coletados foram submetidos aos testes de significância estatística, apresentando comportamento adequado para normalidade, homocedasticidade e homogeneidade. Foi considerado um valor-p significativo acima de 0,050, ou seja, 95\% de significância.

\section{RESULTADOS}

O resultado (nota) obtido por cada estudante que participou do projeto elétrico da proposta de intervenção na entrega final pode ser visto no Quadro 2. Ressalta-se que a turma finalizou com 23 estudantes cursando a disciplina. 
Quadro 2 - Notas finais dos estudantes da CIV0212

\begin{tabular}{|c|c|c|c|c|c|}
\hline Estudante & Grupo & Nota & Estudante & Grupo & Nota \\
\hline 1 & B & 5,93 & 13 & B & 8,28 \\
\hline 2 & B & 6,41 & 14 & A & 9,48 \\
\hline 3 & B & 5,93 & 15 & A & 8,38 \\
\hline 4 & B & 9,11 & 16 & A & 9,48 \\
\hline 5 & B & 5,84 & 17 & A & 9,48 \\
\hline 6 & B & 7,25 & 18 & A & 8,68 \\
\hline 7 & B & 7,25 & 19 & A & 8,63 \\
\hline 8 & B & 6,41 & 20 & A & 3,39 \\
\hline 9 & B & 6,41 & 21 & A & 8,68 \\
\hline 10 & B & 9,11 & 22 & A & 8,63 \\
\hline 11 & B & 5,84 & 23 & A & 8,63 \\
\hline 12 & B & 8,28 & - & - & - \\
\hline
\end{tabular}

Fonte: Autores (2020)

Os seguintes elementos do modelo CANVAS foram preenchidos pelos alunos da disciplina de Projeto de Instalações Elétricas Prediais: Justificativa, Objetivo Smart, Benefícios, Produto Final, Requisitos do Produto, Stakeholders, Equipe, Restrições, Premissas, Grupo de entregas e Riscos. O Quadro 3 mostra um resumo sobre o nível de entendimento (Fácil, Médio e Difícil) desses elementos frente a análise dos alunos da turma de Gerenciamento de Projetos.

Quadro 3 - Níveis empregados para análise do CANVAS

\begin{tabular}{|c|c|c|c|}
\hline Nível de Entendimento & Fácil & Médio & Difícil \\
\hline & Objetivo & & Premissas \\
Elementos do CANVAS & Produto final & Justificativa & Restrições \\
& Stakeholders & Benefícios & Riscos \\
\hline
\end{tabular}

Fonte: Autores (2020)

Assim, considera-se os elementos com nível de entendimento Fácil os elementos do CANVAS onde todas as quatro equipes de Projetos de Instalações Elétricas Prediais definiram corretamente os itens, não havendo discordância sobre isso, por parte das seis equipes avaliadoras de Gerenciamento de Projetos. De certa forma estes quatro elementos estão explícitos no projeto da disciplina, minimizando dessa forma a falta de compreensão.

Os elementos onde o entendimento foi de entendimento Médio dizem respeito aos que não houve consenso nas respostas. Especificamente com relação a justificativa do projeto não ficou claro para metade das equipes o que seria a mesma. Com relação aos requisitos do produto final, apesar dos requisitos da entrega do produto estarem bem definidos na proposta do projeto da disciplina, os alunos ficaram confusos sobre a definição desse item, mostrando que de alguma forma os requisitos do projeto não eram óbvios para eles.

Por último, os elementos considerados de Difícil entendimento foram aqueles em que as equipes da disciplina de Projeto de Instalações Prediais não souberam responder de forma correta. Esses elementos foram as premissas, restrições e riscos. Trata-se de elementos de maior dificuldade de compreensão. Desta forma, riscos e restrições associadas ao tempo disponível da equipe para o desenvolvimento do projeto foram considerados, o que estaria correto. Contudo, a condução da disciplina de projetos foca muito mais no projeto fim, criando dúvidas se riscos, restrições e premissas não deveriam estar associados somente a esses elementos.

O resultado das análises estatística está apresentado conforme cada equipe: 
a) equipe 01: a ANOVA apresentou o valor de $\mathrm{p}=0,038$, ou seja, indicou que há diferença da nota recebida pela primeira entrega entre os estudantes que receberam orientação CANVAS dos estudantes que não receberam. A média das notas foram menores entre os estudantes que receberam a orientação sobre o CANVAS. Os estudantes que receberam a orientação e afirmaram que a orientação foi útil obtiveram uma média de desempenho da nota maior do que os estudantes que receberam a orientação e afirmaram que não foi útil. As médias das notas foram maiores entre os estudantes que estão no $8^{\mathrm{a}}$ semestre ou superior. O curso que o estudante está matriculado não influenciou na nota do estudante. A orientação do CANVAS foi percebida como sendo positiva pelos estudantes que estão nos semestres iniciais do curso. De forma geral observa-se que, quanto maior o grau de envolvimento do grupo (questão do questionário), maior a média das notas obtidas (avaliadas pelo professor na primeira entrega);

b) equipe 02: com 95\% de confiança, há diferença nas notas dos alunos que receberam orientação com relação aos que não receberam orientação CANVAS;

c) equipe 03: o valor de $\mathrm{R}^{2}$ apresentado foi de 0,734 , ou seja, a variação das notas obtidas pelos estudantes na disciplina de Projeto Elétrico foi 73,4\% explicada pelo modelo de regressão aplicado. Ao analisar a equação regressora, encontram-se as variáveis "dedicação no desenvolvimento do projeto", "recebeu orientação" e, "cumprimento dos prazos" como as que mais explicaram a variável y;

d) equipe 04: a análise fatorial foi realizada contemplando as cinco variáveis que obtiveram o índice mais elevado na análise de regressão múltipla. O resultado do teste aponta que há diferença significativa nas notas dos estudantes em função da orientação recebida e, nas demais variáveis, não há diferença significativa entre os grupos. As variáveis investigadas foram: dedicação no desenvolvimento do projeto; recebeu orientação; cumprimento dos prazos; eficácia da comunicação entre os membros da equipe; e, tempo adequado para tarefa;

e) equipe 05: o modelo regressor gerado foi significativo e encontrou o coeficiente de determinação $\mathrm{R}^{2}$ de 0,978 , ou seja, 97,8\% dos dados adequaram-se ao modelo. As variáveis que mais explicam o comportamento da regressora (aprendi os conteúdos da disciplina) são "o projeto entregue atende a qualidade determinada pelo professor" e "a comunicação dos membros da equipe ocorreu de forma eficaz".

É possível observar que havia uma distribuição uniforme dos estudantes em relação ao semestre que eles estão no curso de graduação; sendo que 91,3\% dos estudantes estavam matriculados no curso de Engenharia Civil. Destacamos que 52,2\% da turma (Grupo B) recebeu orientação do CANVAS para escopo do projeto enquanto $47,8 \%$ não recebeu (Grupo A). Metade dos estudantes que receberam orientação afirmam que a orientação auxiliou para a primeira entrega do projeto e outra metade disse que não fez diferença. Destaca-se que 91,3\% dos estudantes afirmam ter aprendido os conteúdos da disciplina, e $87 \%$ sentem-se aptos a aplicar o conhecimento adquirido em outros projetos similares.

\section{CONSIDERAÇÕES FINAIS}

Esse trabalho apresentou o resultado de uma proposta de intervenção interdisciplinar para verificar a possível diferença no resultado de aprendizagem obtido por estudantes, considerando orientações de conteúdos complementares para o planejamento do escopo de projetos. Os resultados da experiência podem ser divididos sob duas óticas.

Numa primeira, pode-se considerar a dinâmica de trabalho entre os docentes das três disciplinas, mostrando que aplicações didáticas de forma interdisciplinar, entre diferentes cursos e com disciplinas de diferentes semestres são possíveis, desde que haja um empenho associado a discussões, planejamento e engajamento por parte dos docentes envolvidos. Isso 
significa, entre outras coisas, uma alta flexibilidade de ajustes na sequência de conteúdos abordados pelas disciplinas, permitindo a sincronização delas.

Outra ótica é com relação aos resultados obtidos pelos estudantes. Estatisticamente, não houve melhoria da nota do grupo que recebeu a orientação por meio da primeira entrega do projeto no modelo CANVAS, ao comparar a nota dos estudantes divididos em dois grupos. Entende-se que essa constatação confirma que as orientações da disciplina são claras e que os estudantes preferem se guiar pelas orientações iniciais do professor da própria disciplina e que, talvez, seja necessário um período maior para explicação e engajamento da turma quanto ao uso da ferramenta CANVAS de projeto. Ainda, o fato de que o modelo da disciplina de Instalações Elétricas Prediais já está direcionado a execução de um projeto elétrico pode indicar que não é necessário uso de um ambiente de apoio. Com relação aos os estudantes das turmas de Gestão de Projetos e Planejamento e Avaliação de Experimentos, percebe-se que houve uma boa receptividade ao se engajaram na proposta intervenção, seja pelo fato de atuarem como críticos do processo, seja pelo fato de atuarem com um caso real. Os estudantes ficavam entusiasmados e discutiam durante as aulas novas possibilidades de intervenção e medição. Na avaliação final, a maior parte dos estudantes se manifestou positivamente pela aplicação da atividade de intervenção interdisciplinar, sendo a percepção coletada por meio de questionário. Como estudo futuro sugere-se a aplicação de outras estratégias para coleta e análise do resultado, além das notas finais, para verificação do nível de aprendizagem obtido pelos alunos.

\section{REFERÊNCIAS}

BAZZO, W. A.; PEREIRA, L. T. do V. Introdução à engenharia: conceitos, ferramentas e comportamentos. 4. ed. rev. Florianópolis: UFSC, 2013.

CAMARGO, C., OLIVEIRA, M. F. de. Painel integrado: envolvendo todos individualmente. In: LEAL, E. A. (org.); MIRANDA, G. J.; CASA NOVA, S. P. C Revolucionando a Sala de Aula: como envolver o estudante aplicando técnicas de metodologias ativas de aprendizagem. São Paulo: Atlas, 2019. p. 187-199

DEMO, P. Conhecimento moderno: sobre ética e intervenção do conhecimento. Petrópolis, RJ: Vozes, 1997.

FAZENDA, I. (Org.). Integração e interdisciplinaridade no ensino brasileiro: efetividade ou ideologia. 6. ed. São Paulo: Loyola, 2011.

FERRARO, J. L. S. Currículo, experimento e experiência: contribuições da Educação em Ciências. Educação, v. 40, n. 1, p. 106-114, jan./abr. 2017.

FINOCCHIO JUNIOR, José. Project Model Canvas: gerenciamento de projetos sem burocracia. Rio de Janeiro: Elsevier, 2013.

FOUCAULT, M. El gobierno de sí y de los otros. Buenos Aires: Fondo de Cultura Económica, 2011.

GIDO, J.; CLEMENTS, J.; BAKER, R. Gestão de projetos. São Paulo: Cengage, 2018.

HUYGHE, S., TOTTÉ, N., VERHAGEN, A. Building the curriculum in Higher Education: a conceptual framework. In: Proceedings of Enhancement and Innovation in Higher Education Conference. Anais..., 2013. p. 11-13. 
"Os desafios para formar hoje o engenheiro do amanhã"

LARROSA, J. Notas sobre a experiência e o saber de experiência. Revista Brasileira de Educação, v. 19, n. 1, 2002.

LÜCK, H. Pedagogia interdisciplinar: fundamentos teórico-metodológicos. 8. ed. Petrópolis: Vozes, 2000.

MADUREIRA, O. M. de. Metodologia de projeto: planejamento, execução e gerenciamento. São Paulo: Blucher, 2010.

PAVIANI, J. Interdisciplinaridade: conceitos e distinções. 2. ed. Caxias do Sul: EDUCS, 2008.

RIBEIRO, L. R. C. ESCRIVÃO-FILHO, E., MIZUKAMI, M. G. N. Uma experiência com PBL no ensino de engenharia sob a ótica dos alunos. Revista de Ensino de Engenharia. v. 23, n. 1, p. 63-80, 2004.

ROSA, K. S., BARCELOS, N. N. Trabalho em grupo: concepções, práticas e contribuições no curso de ciências biológicas. In: ENCONTRO NACIONAL DE PESQUISA EM EDUCAÇÃO EM CIÊNCIAS. Anais... Baurú, Abrapec, 2005.

SOUZA, L.N., CASA NOVA, S.P.C. O role-play (jogo de papéis) aplicado no ensino e aprendizagem. In: LEAL, E. A. (org.); MIRANDA, G. J.; CASA NOVA, S. P. C

Revolucionando a Sala de Aula: como envolver o estudante aplicando técnicas de metodologias ativas de aprendizagem. São Paulo: Atlas, 2019. p. 153-168.

\title{
AN INTERDISCIPLINARY APPROACH FOR ENGINEERING EDUCATION: REPORTING AN EXPERIENCE
}

\begin{abstract}
This article presents the result of an intervention work on the interdisciplinary teaching process in classes of engineering. The work was developed through a case study carried out in three disciplines, involving the following engineering carries: production, control and automation, civil, electrical and in the architecture. An exploratory and descriptive approach with a qualitative character was used. The main theme of the study was the development of an electrical project, guided by the concepts of project management and with an analysis of experiments as a way to evaluate the results of the work. 23 students from the Electrical Projects course, 28 students from the Project Management course and 22 students from the Experiment Design course were involved. The results showed that the integration of contents within different courses, which go beyond the traditional boundaries of undergraduate courses, is possible. As result from the students involved in this intervention project, they signaled positively for the opportunity to apply the concepts developed in actual scenarios of other classes in the same semester. Future studies are necessary in order to stratify the sample in more detail to assess the level of assimilation, through the results, of the students regarding the orientations given, which were pre-established in the experiment. Finally, it is necessary to confirm whether or not there is a need for a longer period for explaining and engaging the class regarding the use of the project CANVAS tool, used in this interdisciplinary intervention.
\end{abstract}

Keywords: Interdisciplinarity. Project. CANVAS. 